\title{
A modified Mediterranean diet is associated with the greatest reduction in alanine aminotransferase levels in obese type 2 diabetes patients: results of a quasi-randomised controlled trial
}

\author{
A. Fraser • R. Abel • D. A. Lawlor • D. Fraser • \\ A. Elhayany
}

Received: 19 December 2007 / Accepted: 24 April 2008/Published online: 3 July 2008

(C) Springer-Verlag 2008

\begin{abstract}
Aim The aim of the study was to compare the effect of different dietary interventions on alanine aminotransferase (ALT) in obese patients with diabetes.

Methods A post hoc analysis of an open label, parallel design, quasi-randomised (allocation by alternation), controlled trial, conducted in Israel. Obese patients with diabetes $(n=259)$, treated in the community, were centrally allocated to one of three diets: (1) the 2003 recommended American Diabetes Association diet (ADA): 50-55\% carbohydrate, $30 \%$ fat and $20 \%$ protein, $n=85$; (2) a low glycaemic index (LGI) diet: 50-55\% LGI carbohydrate, $30 \%$ fat, $15-20 \%$ protein, $n=89$; or (3) a modified Mediterranean diet (MMD): 35\% LGI carbohydrate, $45 \%$ fat that was high in monounsaturated fat, $15-20 \%$ protein, $n=85$. ALT was measured at 6 and 12 months.

Results ALT levels decreased in all arms; however, the MMD was associated with the lowest ALT levels at month $6(n=201$ : ADA $n=64$, LGI $n=73$, MMD $n=64$ ) and month 12 of
\end{abstract}

A. Fraser $(\bowtie) \cdot$ D. A. Lawlor

Department of Social Medicine, University of Bristol,

Canynge Hall, Whiteladies Road,

Bristol BS8 2PR, UK

e-mail: Abigail.fraser@bristol.ac.uk

R. Abel $\cdot$ A. Elhayany

Clalit Health Services, Central District,

Rishon Lezion, Israel

\section{Fraser}

Epidemiology Department and S. Daniel Abraham International

Center for Health and Nutrition,

Ben Gurion University of the Negev,

Beer-Sheva, Israel follow-up ( $n=179)$. At 12 months mean ALT levels were $19.8 \pm 1.4 \mathrm{U} / 1$ in the ADA diet arm $(n=54), 18.0 \pm 1.5 \mathrm{U} / 1$ in the LGI diet arm $(n=64)$ and $14.4 \pm 1.7$ in the MMD arm $(n=61, p<0.001)$. Evidence for an effect of diet on ALT levels persisted when controlling for post-randomisation changes in waist to hip ratio, BMI, homeostasis model assessment (HOMA) or triacylglycerol.

Conclusions A Mediterranean diet may have a beneficial effect on liver steatosis in obese patients with diabetes. Results of trials assessing the effect of dietary composition on clinical outcomes should be awaited before a decisive conclusion can be reached. In addition to clinical outcomes, such studies should address the issue of primary prevention of steatosis in high-risk and healthy individuals.

\section{Trial registration: ClinicalTrials.gov NCT00520182}

Funding: This study was supported by a grant from Tnuva Research Institute, Rehovot, Israel.

Keywords Alanine aminotransferase - Mediterranean diet . Non-alcoholic fatty liver disease $\cdot$ Randomised controlled trial - Type 2 diabetes

$\begin{array}{ll}\text { Abbreviations } \\ \text { ADA } & \text { American Diabetes Association } \\ \text { ALT } & \text { alanine aminotransferase } \\ \text { HDL-c } & \text { HDL-cholesterol } \\ \text { HOMA } & \text { homeostasis model assessment } \\ \text { LGI } & \text { low glycaemic index } \\ \text { MMD } & \text { modified Mediterranean diet } \\ \text { NAFLD } & \text { non-alcoholic fatty liver disease } \\ \text { NASH } & \text { non-alcoholic steatohepatitis }\end{array}$




\section{Introduction}

Non-alcoholic fatty liver disease (NAFLD) consists of a range of liver abnormalities in the absence of other established causes of liver damage. In its mildest form (steatosis) it is characterised by an accumulation of triacylglycerol in hepatocytes, and its more advanced form, non-alcoholic steatohepatitis (NASH) is characterised by hepatocellular injury and inflammation [1]. NAFLD has been termed the hepatic manifestation of the insulin resistance syndrome due to its close associations with obesity, insulin resistance and type 2 diabetes. It has been estimated that up to $70 \%$ of patients with type 2 diabetes also have NAFLD [2].

Accumulation of fat in the liver is caused by increased delivery of non-esterified fatty acids to the liver originating from dietary fat intake and adipose tissue, as well as hepatic synthesis. In addition, impaired non-esterified fatty acid oxidation and export of triacylglycerol from the liver contribute to accumulation of fat in the liver [1]. Dietary intake plays a role in liver fat accumulation, both directly and indirectly (via its influence on adipose tissues). It has been estimated that dietary sources account for $15 \%$ of liver fat content [3].

Currently, there is no evidence-based established treatment for NAFLD, but weight loss is recommended for overweight NAFLD patients. The American Gastroenterological Association dietary recommendations also include energy restriction, a decrease in saturated fats as well as total fat to $<30 \%$ of total energy, and an increase in fibre intake, while recognising that such recommendations remain unsupported by robust evidence [4]. These uncertainties have led to an increasing interest in the possible role of dietary composition in both the prevention and treatment of NAFLD [5].

We conducted a post hoc analysis of a quasi-randomised controlled trial to determine the effect of three different dietary interventions on alanine aminotransferase (ALT) in obese, type 2 diabetes patients. ALT is used as a screening and initial diagnostic marker for NAFLD in clinical settings $[4,6]$ and as a biomarker of NAFLD and liver fat content in epidemiological studies in which the use of more accurate but invasive and/or expensive tests is unethical or not feasible.

\section{Methods}

The trial design and methods have been presented in detail elsewhere [7]. Briefly, 259 eligible type 2 diabetes patients were recruited between March 2003 and April 2004 from ten urban primary care clinics, using the electronic database of the central region of the largest health maintenance organisation in Israel (Clalit Health Services). Inclusion criteria were: (1) type 2 diabetes diagnosed within the last 10 years; (2) 30-65 years of age; (3) BMI between 27 and $34 \mathrm{~kg} / \mathrm{m}^{2}$; (4) $\mathrm{HbA}_{1 \mathrm{c}}$ levels 7-10\%; (5) plasma triacylglycerol level 1.8-4.5 mmol/l; (6) creatinine $<123.2 \mu \mathrm{mol} / \mathrm{l}$; and $(7) \geq 3$ months on the same medication. Exclusion criteria were: (1) proliferative diabetic retinopathy; (2) current insulin treatment; (3) active oncological or psychiatric disease; and (4) uncontrolled hypothyroidism or hyperthyroidism.

We identified 2,486 type 2 diabetes patients, 1,063 $(42.8 \%)$ of whom met the inclusion criteria, and 644 $(60.6 \%)$ of those were willing to participate. Before study entry, patients were asked to repeat laboratory tests and to undergo fundoscopy: 244 patients did not complete the tests and an additional 141 were excluded due to new findings, thus 259 patients were recruited. The trial flow is presented in Fig. 1.

Intervention Patients were recruited to the trial by the study coordinator (R. Abel). Prior to randomisation, participants entered a 2 week maintenance period, during which they continued their usual diet and kept a food diary. An external person, who had no involvement in participant recruitment and had no knowledge of any participant characteristics, then allocated patients to the different trial arms and participating dietitians, using a systematic sequence. Although a systematic sequence rather than randomisation was used, concealment of allocation was protected, i.e. neither the recruiter nor the potential participant could foresee to which arm the potential participant would be allocated, as allocation was performed centrally and both the potential participant and recruiter were blinded to the allocation procedure and its outcome. The allocation of patients to different dietitians ensured the equal distribution of patients assigned to the three different diets between participating dietitians.

The three dietary interventions were similar in total energy intake but varied in fat and carbohydrate constituents as follows: (1) the 2003 recommended American Diabetes Association diet (ADA) [8] (50-55\% carbohydrate of mixed glycaemic index, $30 \%$ fat and $15-20 \%$ protein); (2) a low glycaemic index (LGI) diet (50-55\% LGI carbohydrate, 30\% fat, $15-20 \%$ protein); (3) a modified Mediterranean diet (MMD) (35\% LGI carbohydrate, $45 \%$ fats high in monounsaturated fat content, $20 \%$ protein). Energy ( $97 \mathrm{~kJ} / \mathrm{kg}$ body weight), sodium (up to $3 \mathrm{~g} /$ day), potassium ( $>3 \mathrm{~g}$ /day), calcium $(\sim 1,300 \mathrm{mg} /$ day $)$ and magnesium $(>800 \mathrm{mg} /$ day $)$ contents were similar in all three diets.

The process of providing dietary advice was identical in all three intervention groups. All three diet plans were based on food groups, and participants could design their own plan, using these groups. The ADA diet included light whole wheat bread, whole rice, bran flakes, rice cakes, fruit, 
Fig. 1 Trial flow diagram. ADA, American Diabetes Association; ALT, alanine aminotransferase; ITT analysis, intention to treat analysis; LGI, low glycaemic diet; MMD, modified Mediterranean diet. The ITT analysis included all randomised patients for whom a baseline ALT measurement was available $(n=259)$. The last available measurement was carried forward

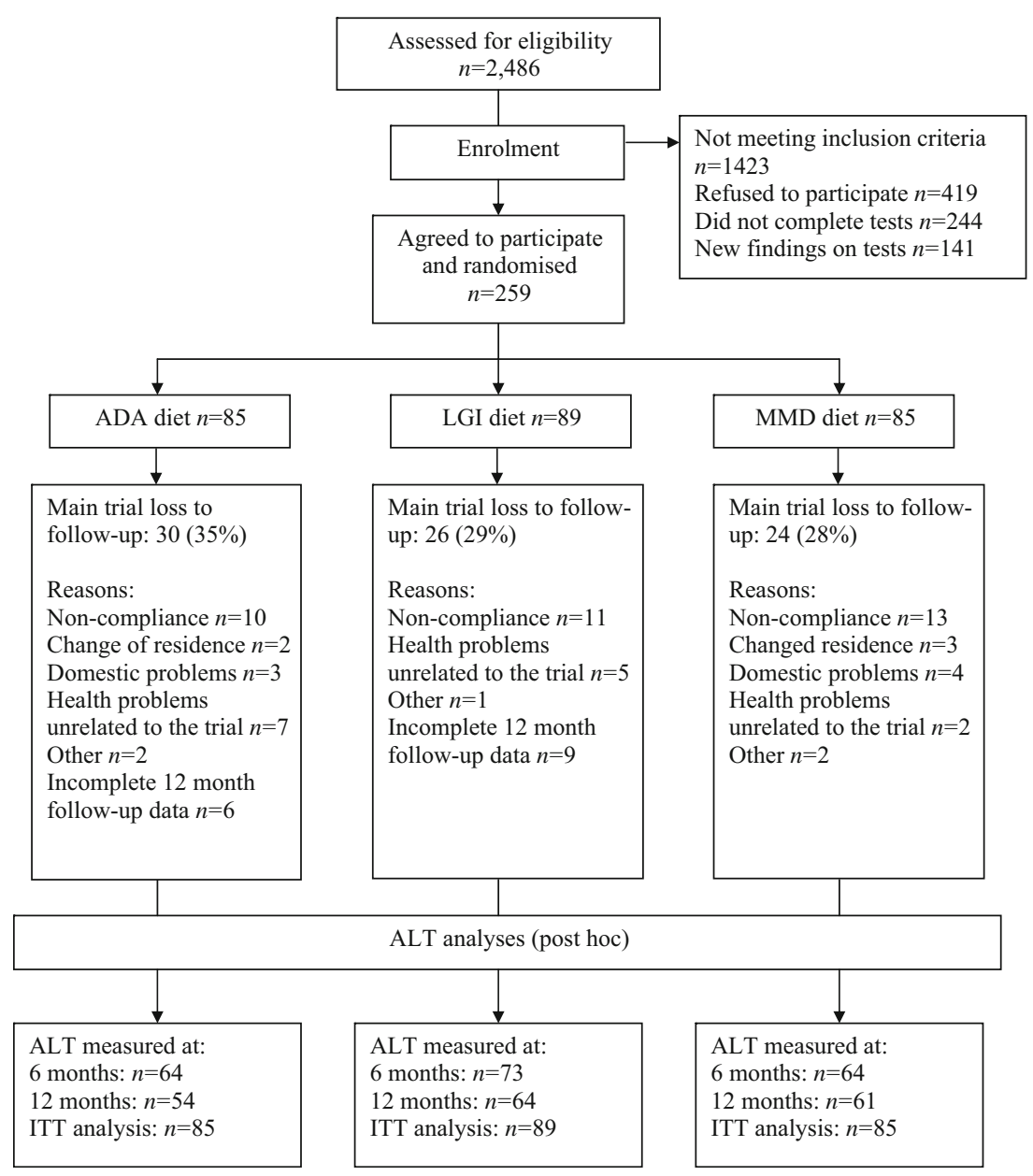

vegetables, low sugar jam and honey. The LGI diet replaced wheat with oats, bread and rice with lentils, chickpeas and white beans. The MMD included fish, replaced butter with olive oil and olive-based margarine, and nuts and almonds. All diets included egg, chicken and turkey breast. All participants were also advised (using the same method of verbal advice in all three treatment arms) to undertake at least $30 \mathrm{~min}$ of aerobic activity three times a week, as this has been shown to have a beneficial effect on glucose tolerance and insulin sensitivity, as well as other cardiovascular risk factors [9]. Participants met with their assigned dietitian every 2 weeks for 12 months for counselling. All dietitians worked according to a structured protocol for the 24 scheduled meetings and supervised patients from all three diets. Every other month, all patients were invited to a group meeting to discuss general health and emotional issues.

At baseline, participants completed a food frequency questionnaire [10, 11], a $24 \mathrm{~h}$ diet recall questionnaire and a physical activity questionnaire in the presence of a dietitian and underwent a medical examination by their treating physician which included taking blood samples after an overnight fast. These procedures were repeated after
6 months of follow-up and again after 12 months, except for the dietary questionnaires that were not administered again after 12 months. All blood tests were performed in a central laboratory of Clalit Health Services using standard procedures: ALT was determined according to the recommendations of the International Federation of Clinical Chemistry (IFCC) using a photometric method without pyridoxal phosphate (Hitachi 747; Indianapolis, IN, USA); fasting plasma glucose was measured by the photometric glucose oxidase technique (Hitachi 747); plasma insulin was measured by the immune-enzymatic technique (Immulite 2000; Siemens Healthcare Diagnostics, Deerfield, IL, USA) that does not cross-react with pro-insulin; total cholesterol and HDL-cholesterol (HDL-c) were measured using a photometric enzymatic method (Hitachi 747); and triacylglycerol using a photometric-glycerol phosphateenzymatic method (Hitachi 747).

Pre-defined trial outcomes were: glycated haemoglobin, fasting plasma glucose, triacylglycerol (primary outcomes), and weight, BMI, waist and hip circumference, HDL-c and insulin resistance as assessed by the homeostasis model assessment (HOMA) [12]. ALT was not measured as part of the trial protocol and was available only for those participants 
whose treating physicians included liver function tests as part of routine testing. It was estimated that 55 participants per arm would be required to detect a $20 \%$ change in triacylglycerol from baseline or a $10 \%$ difference between trial arms with $80 \%$ power and a $5 \%$ significance level.

All participants provided written informed consent. The study protocol was approved by the ethics committee of Soroka University Medical Center, Ben Gurion University, Beer Sheva, Israel.

Statistical analysis ALT, fasting insulin, HOMA and triacylglycerol values had skewed distributions and were normalised using a natural-log transformation. The effects of the different diets on ALT levels at 6 and 12 months were examined both by comparing ALT levels between groups while controlling for baseline ALT using analysis of covariance, and by comparing ALT at 6 and 12 months to baseline ALT levels within groups (by a paired $t$ test).

For the main analysis we included participants for whom both baseline and follow-up (at 6 or 12 months) measurements were available $(n=201,78 \%)$. We also conducted a secondary analysis using a full intention to treat approach. This analysis included all randomised patients $(n=259)$. In this analysis the last available measurement was carried forward. In other words, for participants for whom ALT measurements at month 12 were missing, the ALT measurement obtained from month 6 was used and if that was unavailable, then the baseline measurement was included.

Finally, multivariable linear regression models were constructed to examine the effect of possible mediators (change between baseline and 6 months in BMI, waist to hip ratio, triacylglycerol and HOMA) on any association between diet and ALT levels at 6 months. All analyses were conducted in Stata version 9 (Stata Corporation, College Station, TX, USA).

\section{Results}

Baseline characteristics of trial participants for whom an ALT result was available at baseline and either at 6 or 12 months of follow-up and therefore included in the main analyses of this report $(n=201)$, are presented in Table 1 . Participant characteristics were similar between study arms whether all patients included in this analysis $(n=201)$ or all randomised participants $(n=259)$ were compared. The 201 patients with ALT measurements had lower fasting glucose measurements ( $10.3 \mathrm{vs} 11.0 \mathrm{mmol} / \mathrm{l}, p=0.03$ ) than the other 58 randomised participants. No other differences were noted. Finally, the proportion of participants lost to follow-up per trial arm at 6 and 12 months were similar.

Dietary intake, as reported in food frequency questionnaires by participants $(n=195)$ at 6 months of follow-up, is presented in Table 2 . These results provide face validity for adherence to the three diets, although differences were smaller than originally planned. Overall, there was no statistically significant difference in mean total energy intake between the three study arms or in the per cent of protein consumed. Mean per cent fat intake was similar in the ADA and LGI arms and higher in the MMD arm. Monounsaturated fat intake was higher in the MMD arm than in the other two arms. Carbohydrate intake was lower in the MMD arm than in the ADA and LGI arms.

Table 1 Patient characteristics at baseline

\begin{tabular}{|c|c|c|c|}
\hline Characteristic & $\operatorname{ADA}(n=64)$ & LGI $(n=73)$ & $\operatorname{MMD}(n=64)$ \\
\hline Male (\%) & $31(48)$ & $40(55)$ & $32(50)$ \\
\hline Age (years) & $55.8(6.2)$ & $57.0(5.9)$ & $55.2(6.8)$ \\
\hline $\operatorname{ALT}(\mathrm{U} / 1)^{\mathrm{a}}$ & $25.0(1.5)$ & $22.8(1.4)$ & $24.5(1.6)$ \\
\hline BMI $\left(\mathrm{kg} / \mathrm{m}^{2}\right)$ & $31.9(3.2)$ & $31.2(2.7)$ & $31.4(2.7)$ \\
\hline Waist $(\mathrm{cm})$ & $113.7(9.8)$ & $111.0(8.9)$ & $112.5(9.8)$ \\
\hline Hip (cm) & $114.1(9.5)$ & $111.7(8.5)$ & $111.6(7.9)$ \\
\hline $\mathrm{HbA}_{1 \mathrm{c}}(\%)$ & $8.4(0.8)$ & $8.3(1.0)$ & $8.4(1.0)$ \\
\hline Fasting glucose (mmol/l) & $10.4(1.7)$ & $10.2(1.9)$ & $10.4(1.9)$ \\
\hline Fasting insulin $\left(\mathrm{pmol} / \mathrm{l}^{\mathrm{a}}\right.$ & $81.3(12.5)$ & $73.6(13.2)$ & $77.1(12.5)$ \\
\hline HOMA score sa $^{\mathrm{a}}$ & $5.4(1.8)$ & $4.7(1.9)$ & $5.9(1.7)$ \\
\hline Total cholesterol (mmol/l) & $5.4(0.9)$ & $5.4(0.8)$ & $5.3(0.9)$ \\
\hline HDL-c $(\mathrm{mmol} / \mathrm{l})$ & $1.1(0.2)$ & $1.1(0.2)$ & $1.1(0.2)$ \\
\hline Triacylglycerol $(\mathrm{mmol} / \mathrm{l})^{\mathrm{a}}$ & $2.6(1.3)$ & $2.6(1.2)$ & $2.7(1.3)$ \\
\hline Systolic blood pressure (mmHg) & $138.4(14.5)$ & $142.3(13.6)$ & $142.2(15.6)$ \\
\hline Daily energy intake $(\mathrm{kJ})^{\mathrm{b}}$ & $14,382(5744)$ & $13,289(5568)$ & $13,733(5832)$ \\
\hline Daily energy expenditure (kJ) & $11,103(1537)$ & $10,748(1729)$ & $10,660(1503)$ \\
\hline
\end{tabular}

Values are means (SD) unless otherwise noted

${ }^{\text {a }}$ Geometric mean $(\mathrm{SD})$

${ }^{\mathrm{b}} \mathrm{ADA}, n=62$; LGI, $n=70$; MMD, $n=63$ 
Table 2 Dietary intake (mean, SD) as reported in the food frequency questionnaires administered at month 6

\begin{tabular}{|c|c|c|c|c|}
\hline Parameter & $\operatorname{ADA}(n=61)$ & LGI $(n=71)$ & $\operatorname{MMD}(n=63)$ & $p$ value \\
\hline Total daily energy intake (kJ) & $8,742(3,395)$ & $8,315(2,692)$ & $9,316(3,768)$ & 0.23 \\
\hline Carbohydrate $(\%)$ & $46(7.1)$ & $45(6.8)$ & $42(7.5)$ & $<0.01^{\mathrm{a}}$ \\
\hline Protein $(\%)$ & $19(2.8)$ & $20(3.3)$ & $19(3.4)$ & 0.22 \\
\hline Fat $(\%)$ & $3,737(6.3)$ & $36(5.6)$ & $41(6.6)$ & $<0.001^{\mathrm{a}}$ \\
\hline Monosaturated fat of total fat (\%) & $34(2.0)$ & $35(2.3)$ & $36(2.4)$ & $0.001^{\mathrm{a}}$ \\
\hline
\end{tabular}

${ }^{a}$ MMD differs from both ADA and LGI, but ADA and LGI do not differ from each other

Results of the comparison of mean ALT levels at months 6 and 12 across trial arms and within trial arms (compared with baseline) are presented in Table 3. ALT levels decreased in all trial arms and were statistically significantly lower (all $p$ values $\leq 0.002$ ) in all of the trial arms at both months 6 and 12, when compared with baseline. When ALT levels at months 6 and 12 were compared across trial arms and adjusting for baseline measurements, the MMD was associated with the lowest mean ALT compared with both the ADA and the LGI diets.

We examined the effect of including possible mediators (changes in BMI, waist to hip ratio, triacylglycerol and HOMA between baseline and follow-up by randomised group) in a multivariable model of ALT levels at month 6, at which there was the least amount of missing data. Results are presented in Table 4. Changes in waist to hip ratio and HOMA were both strongly associated with ALT levels at month 6. However, including waist to hip ratio (Table 4, model 3) or HOMA (Table 4, model 5) in the model did not attenuate the association between diet and ALT levels. In the model including baseline ALT and changes in waist to hip ratio, triacylglycerol and HOMA, the ratio of geometric means of ALT in the LGI arm versus the ADA arm was $1.06(95 \%$ CI $0.95-1.21, p=0.35)$ and in the MMD versus the ADA arm it was 0.77 (95\% CI $0.68-0.88, p<0.001)$. When we repeated the analysis using ALT measured at 12 months as the outcome, the results were essentially the same as those presented in Table 4 (results not shown).

Table 3 Geometric means (SD) of alanine aminotransferase (ALT) $(\mathrm{U} / 1)$ at baseline, 6 and 12 months

\begin{tabular}{llllc}
\hline Parameter & $\begin{array}{l}\text { ADA } \\
(n=64)\end{array}$ & $\begin{array}{l}\text { LGI } \\
(n=73)\end{array}$ & $\begin{array}{l}\text { MMD } \\
(n=64)\end{array}$ & p value \\
\hline Baseline & $25.0(1.5)$ & $22.8(1.4)$ & $24.5(1.6)$ & 0.41 \\
6 months & $19.4(1.6)$ & $18.8(1.6)$ & $14.7(1.6)$ & $<0.001^{\mathrm{a}, \mathrm{b}}$ \\
12 months $^{\mathrm{c}}$ & $19.8(1.4)$ & $18.0(1.5)$ & $14.4(1.7)$ & $<0.001^{\mathrm{a}, \mathrm{b}}$ \\
\hline
\end{tabular}

${ }^{a}$ MMD was different from both ADA and LGI. The latter were not different from each other

${ }^{\mathrm{b}} p$ value adjusted for baseline measurement

${ }^{c}$ At 12 months ALT measures were available for 179 participants.

ADA, $n=54$; LGI, $n=64$; MMD, $n=61$
In the secondary, intention to treat analysis conducted by carrying the last result forward and including all randomised patients $(n=259)$, mean ALT in the MMD arm (16.4 U/l) was lower when compared with the ADA diet (22.7 U/1), with mean ALT levels in the LGI arm (18.7 U/l) falling between these two $(p<0.001)$.

\section{Discussion}

In this quasi-randomised controlled trial, a modified Mediterranean diet, which is relatively high in fat content (but with most of the fat content from monounsaturated fats), was associated with lower ALT levels at both 6 and 12 months compared with both the 2003 ADA diet and a LGI diet. While the ADA and the LGI diets contain the same percentage of carbohydrates, the LGI diet includes carbohydrates of low (as opposed to mixed) glycaemic index. Mean ALT levels were similar at 6 and 12 months in participants allocated to either the LGI or the ADA diet in the main analysis using complete data. In the secondary, intention to treat analysis, in which the last ALT measurement was carried forward, the MMD was still associated with the greatest benefit; however, the LGI diet was also better in reducing ALT than the ADA diet.

Table 4 Ratios of geometric means $(95 \% \mathrm{CI})$ and $p$ values of ALT at 6 months $(n=201)$

Model $\begin{array}{ll}\text { ADA } \\ (n=64)\end{array} \quad$ LGI $(n=73) \quad$ MMD $(n=64)$

\begin{tabular}{llll}
\hline 1 & 1 & $1.04(0.92-1.17), 0.54$ & $0.77(0.68-0.88),<0.001$ \\
2 & 1 & $1.03(0.91-1.16), 0.61$ & $0.78(0.69-0.88),<0.001$ \\
3 & 1 & $1.06(0.94-1.20), 0.35$ & $0.79(0.59-0.90),<0.001$ \\
4 & 1 & $1.04(0.92-1.17), 0.55$ & $0.77(0.68-0.88),<0.001$ \\
5 & 1 & $1.04(0.93-1.18), 0.48$ & $0.76(0.67-0.86),<0.001$ \\
6 & 1 & $1.06(0.95-1.21), 0.35$ & $0.77(0.68-0.88),<0.001$ \\
\hline
\end{tabular}

Model 1, adjusted for: baseline ALT

Model 2, model $1+$ change in BMI from baseline

Model 3, model $1+$ change in waist to hip ratio from baseline, $n=192$ Model 4, model $1+$ change in triacylglycerol from baseline

Model 5, model $1+$ change in HOMA from baseline, $n=197$

Model 6 , model $1+$ change in waist to hip ratio, triacylglycerol and HOMA from baseline, $n=188$ 
Our results are similar to those found in a smaller trial of shorter duration which included 52 obese, insulin resistant participants [13]. In that trial, the effects of two diets with similar energy but different carbohydrate and fat content were compared: one containing $60 \%$ carbohydrate and $25 \%$ fat and the other $40 \%$ carbohydrate and $45 \%$ fat. After 16 weeks of dieting and 2 weeks of weight maintenance, mean weight loss was moderately greater in the lower carbohydrate/higher fat group (7.0 vs $5.7 \mathrm{~kg})$ but this difference did not reach conventional levels of statistical significance. Mean ALT was reduced by $9.5 \mathrm{U} / 1$ in the lower carbohydrate/higher fat group compared with only $4.2 \mathrm{U} / 1$ in the higher carbohydrate/lower fat arm. In an additional study of 25 non-obese, non-diabetic NASH patients and 25 age, sex and BMI matched controls, cases were found to have a significantly lower fibre and polyunsaturated fat intake and higher protein and saturated fat intake [14].

The Mediterranean diet has been reported to have a beneficial effect on other cardiovascular risk factors. Analysis of a subgroup of 772 participants in the PREDIMED study, a large trial including individuals at high risk of cardiovascular disease, demonstrated a beneficial effect of a Mediterranean diet on lipid profiles, insulin resistance, blood pressure and inflammatory markers compared with a low fat diet [15]. In a smaller trial $(n=$ 180 ) with longer follow up (2 years), the Mediterranean diet was found to be more effective for reducing weight, BMI, waist circumference, inflammatory markers, glucose, total cholesterol, triacylglycerol, and insulin resistance, increasing HDL-c, improving endothelial function, and reducing the prevalence of the metabolic syndrome compared with the low fat control diet [16]. Taken together, these results suggest that relatively high dietary fat intake, in itself, is probably not associated with accumulation of fat in the liver or other cardiovascular risk factors, and that the type of dietary fat (saturated fat, monounsaturated fat and polyunsaturated fat) may be more important than the amount.

We hypothesised that weight reduction and/or changes in triacylglycerol levels and/or insulin resistance (as measured by HOMA), may account for the observed association between type of diet and ALT levels. Although decreases in baseline waist to hip ratio and HOMA were associated with ALT levels measure at 6 months, they did not explain the association between diet and ALT. This suggests that dietary intake affects liver fat content by additional pathways to those mediated by adiposity and insulin resistance.

Study strengths include the relatively long-term intervention and follow-up and the monitoring of dietary intake throughout the trial by $24 \mathrm{~h}$ recall questionnaires and food frequency questionnaires. These data enabled us to demonstrate that variation in usual diet pre-randomisation (assessed by baseline measurements) was similar in the three trial arms (Table 1) and provided evidence of adherence to the different diets by participant in the three trial arms, since follow-up measurements indicated anticipated similarities in total caloric intake and per cent protein intake and anticipated differences in carbohydrate and fat type and quantity (Table 2). However, differences were smaller than originally planned. Therefore it is possible that differences in ALT levels would be even greater if more pronounced differences in dietary composition could be achieved.

Some limitations should also be noted. ALT was not a predefined outcome in this trial, which was powered to detect differences in other outcomes and because ALT was not a pre-defined outcome, information was not available for all trial participants. However, a baseline ALT measurement was available for all but one participant and when the last observation was carried forward in the intention to treat analysis, results were not substantially changed from those obtained in the main analysis. Carrying the last observation forward is a very conservative technique that probably leads to underestimated differences between trial arms.

Another limitation is the use of ALT as a biomarker of liver fat, which is only moderately correlated with liver fat content (correlation coefficients ranging from 0.4 [17] to 0.6 [18]) and not a more direct measure of liver fat such as that obtained from proton magnetic resonance spectroscopy.

This trial, like many others, has focused on established cardiovascular risk factors as outcomes and larger trials with cardiovascular events and survival outcomes are required to determine the full extent to which dietary interventions will affect clinical endpoints. Final results of the PREDIMED trial [15] will help in answering this question. With increasing concerns about NAFLD future trials of the effect of dietary interventions on the progression and prevention of NAFLD that use better techniques for diagnosing NAFLD (i.e. magnetic resonance imaging spectroscopy) would also be valuable.

In conclusion, the MMD diet, which is relatively low in carbohydrates and high in monounsaturated fat intake is associated with a greater reduction in ALT levels compared with the recommended ADA diet (2003) or a LGI diet. These differences were not mediated by weight loss, or decreases in HOMA or triacylglycerol levels. These results suggest that a Mediterranean diet may have a beneficial effect on liver steatosis in obese patients with type 2 diabetes. Results of trials assessing the effect of dietary composition on clinical outcomes (such as the PREDIMED trial) should be awaited before a decisive conclusion can be reached. As well as clinical outcomes such as cardiovascular events, such studies should address the issue of primary prevention of liver steatosis both in high-risk and in healthy individuals.

Acknowledgements This study was supported by a grant from Tnuva Research Institute, Rehovot, Israel. A. Fraser receives support from the University of Bristol Overseas Research Scholarship Award 
Scheme and D. Lawlor is funded by a (UK) Department of Health Career Scientist award. We thank all trial participants and dietitians.

Duality of interest The authors declare that there is no duality of interest associated with this manuscript.

\section{References}

1. Harrison SA, Day CP (2007) Benefits of lifestyle modification in NAFLD. Gut 56:1760-1769

2. Targher G, Bertolini L, Padovani R et al (2007) Prevalence of nonalcoholic fatty liver disease and its association with cardiovascular disease among type 2 diabetic patients. Diabetes Care 30:1212-1218

3. Donnelly KL, Smith CI, Schwarzenberg SJ, Jessurun J, Boldt MD, Parks EJ (2005) Sources of fatty acids stored in liver and secreted via lipoproteins in patients with nonalcoholic fatty liver disease. J Clin Invest 115:1343-1351

4. American Gastroenterological Association (2002) American Gastroenterological Association medical position statement: nonalcoholic fatty liver disease. Gastroenterology 123:1702-1704

5. Zivkovic AM, German JB, Sanyal AJ (2007) Comparative review of diets for the metabolic syndrome: implications for nonalcoholic fatty liver disease. Am J Clin Nutr 86:285-300

6. Grant A, Neuberger J, Day C, Saxseena S. British Society for Gastroenterology: Guidelines on the use of Liver Biopsy in Clinical Practice. Available at http://www.bsg.org.uk/pdf_word docs/liver_biopsy.pdf, accessed 1 October 2007

7. Shahar DR, Abel R, Elhayany A, Vardi H, Fraser D (2007) Does dairy calcium intake enhance weight loss among overweight diabetic patients? Diabetes Care 30:485-489

8. Franz MJ, Bantle JP, Beebe CA, et al (2003) Evidence-based nutrition principles and recommendations for the treatment and prevention of diabetes and related complications. Diabetes Care 26:S51-S61
9. Albright A, Franz M, Hornsby G et al (2000) American College of Sports Medicine position stand. Exercise and type 2 diabetes. Med Sci Sports Exerc 32:1345-1360

10. Shahar D, Shai I, Vardi H, Brener-Azrad A, Fraser D (2003) Development of a semi-quantitative food frequency questionnaire (FFQ) to assess dietary intake of multiethnic populations. Eur J Epidemiol 18:855-861

11. Shai I, Rosner BA, Shahar DR et al (2005) Dietary evaluation and attenuation of relative risk: multiple comparisons between blood and urinary biomarkers, food frequency, and 24-hour recall questionnaires: the DEARR study. J Nutr 135:573-579

12. Matthews DR, Hosker JP, Rudenski AS, Naylor BA, Treacher DF, Turner RC (1985) Homeostasis model assessment: insulin resistance and beta-cell function from fasting plasma glucose and insulin concentrations in man. Diabetologia 28:412-419

13. Ryan MC, Abbasi F, Lamendola C, Carter S, McLaughlin TL (2007) Serum alanine aminotransferase levels decrease further with carbohydrate than fat restriction in insulin resistant adults. Diabetes Care 30:1075-1080

14. Musso G, Gambino R, De MF et al (2003) Dietary habits and their relations to insulin resistance and postprandial lipemia in nonalcoholic steatohepatitis. Hepatology 37:909-916

15. Estruch R, Martinez-Gonzalez MA, Corella D et al (2006) Effects of a Mediterranean-style diet on cardiovascular risk factors: a randomized trial. Ann Intern Med 145:1-11

16. Esposito K, Marfella R, Ciotola M et al (2004) Effect of a mediterranean-style diet on endothelial dysfunction and markers of vascular inflammation in the metabolic syndrome: a randomized trial. JAMA 292:1440-1446

17. Tiikkainen M, Bergholm R, Vehkavaara S et al (2003) Effects of identical weight loss on body composition and features of insulin resistance in obese women with high and low liver fat content. Diabetes 52:701-707

18. Westerbacka J, Corner A, Tiikkainen M et al (2004) Women and men have similar amounts of liver and intra-abdominal fat, despite more subcutaneous fat in women: implications for sex differences in markers of cardiovascular risk. Diabetologia 47:1360-1369 